\title{
Non Government Organizations (NGO)-The gap fillers in oncology care during COVID-19 lockdown in Assam, India
}

\author{
Joyeeta Talukdar', Abhibroto Karmakar², Ruby Dhar ${ }^{3}$, Arun Kumar', \\ Subhradip Karmakar ${ }^{5}$ \\ ${ }^{1}$ Research Associate-I, Lab No-3018 Department of Biochemistry, All India Institute of Medical Sciences, New Delhi, \\ India, ${ }^{2}$ Junior Research Fellow, Lab No-3018 Department of Biochemistry, All India Institute of Medical Sciences, New \\ Delhi, India, ${ }^{3}$ Scientist, Room No-3020 Department of Biochemistry, All India Institute of Medical Sciences, New Delhi, \\ India, ${ }^{4}$ Professor and Head, Department of Biochemistry, Jagannath Gupta Institute of Medical Sciences and Hospital, \\ Budge Budge, Kolkata, India, ${ }^{5}$ Associate-Professor, Room No-3020 Department of Biochemistry, All India Institute of \\ Medical Sciences, New Delhi, India
}

A B S T R A C T

COVID-19 pandemic caused due to SARS-CoV2 has disrupted the spectrum of health care. The wide arrays of supportive and palliative care needed for patients with ailments were grossly neglected, due to the diversion of resources and healthcare professionals in COVID management. This deficiency was further exaggerated by extended lockdown and closure of outdoor patient care services resulting in unprecedented crisis in disease management. Further, COVID-19 has been associated with increased risk of morbidity and mortality arising from associated risk factors in geriatric subjects and those with other high risk co-morbidities like hypertension, COPD, diabetes and cancer. North East Indian states and the adjoining regions seem to have suffered substantially during the COVID-19 crisis due to their preexisting vulnerabilities and under developed health care infrastructure and logistics. This deficiency, however, seems to have been fulfilled substantially by the participation of NGO (Non-Government Organizations) and other volunteer services who has actively participated to provide basic healthcare and other life support to cancer patients in this crisis.

Key words: Cancer; Assam; Non-Government Organizations; Covid-19; anxiety; depression

\section{Access this article online}

Website:

http://nepjol.info/index.php/AJMS DOI: $10.3126 /$ ajms.v12i6.36488 E-ISSN: 2091-0576 P-ISSN: $2467-9100$

Copyright (c) 2021 Asian Journal of Medical Sciences

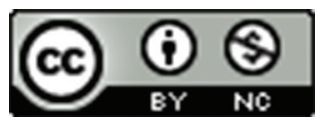

This work is licensed under a Creative Commons Attribution-NonCommercial 4.0 International License.

\section{INTRODUCTION}

North east has been a hub of outgrowing Cancer patients, and Outbreak of Covid-19 has brought the mixed association of psychological distress and the fear of exposure to COVID-19 in Cancer Patients..$^{1-6}$

COVID-19 caused due to Severe Acute Respiratory Syndrome-CoronaVirus-2 (SARS-CoV-2), a member of beta Corona virus has become a worldwide threat and a major healthcare concern. ${ }^{7}$ It has proved to be a pandemic by affecting over 6.9 million people and claiming more than
400,000 lives in over 200 nations worldwide. ${ }^{8}$ Several cohort studies suggested that patients with active malignancy or history of it might be at increased risk of engaging to COVID-19 infections and complications precipitating to medical emergencies (intensive care unit admission, invasive ventilation, ARDS* and death). ${ }^{4-6,9}$

India with a population of more than 1.3 billion people turned out to be the new epicenter of COVID-19. ${ }^{8}$ Nine percent of deaths in India have been reported due to Cancer. ${ }^{10}$ As such, this selected group was in highest risk of being infected during COVID-19 pandemic. 
The projected national cancer incidence burden in 2020 for India estimated to be 98.7 per 100,000 populations with highest being observed in the North eastern region (6 PBCRs* for males and 4 PBCRs for females). ${ }^{11}$ This percentage of increase was almost 7 fold in comparison to the patients registered during NCRP (2006-08) for NE, as described in Table $1 .^{11,12}$ The reasons for this highest number although unclear a complex combination of several factors, including food habitats, lifestyle, occupation, and genetic predisposition seems to be responsible for the high prevalence. ${ }^{1-3}$ Lack of the required specialized infrastructure with multilevel- primary, secondary and tertiary approach is also one of the reasons for low survival rate with higher proportion of recurrence and distant metastasis. ${ }^{13}$ This has initiated the co-dependency of the patients to other states with multidisciplinary facilities that included travelling substantial proportion of cancer patients from NE. ${ }^{14,15}$

With no vaccination or therapeutic drugs, Nonpharmaceutical interventions (NPIs) were aimed at reducing contact rates in population during COVID-19. ${ }^{16,17}$ As such, lockdown was imposed with the concept of "mitigation" for implementing social distancing and preventing community transmissions of the virus in India. ${ }^{18}$ However, the traditional oncology treatment significantly disrupted this social distancing strategy since it involved engagement of continuous clinical visits surgical incisions and stays, sessions of infusion, radiation planning phlebotomy visits for laboratory tests, and radiographic imaging studies which required planned hospital visits accompanied by family members. ${ }^{19}$ Although necessary, this sudden lockdown preceded with psychological effects - post-traumatic stress symptoms, anger, and confusion. The situation worsened for cancer patients with their uncertainty of irregularity in their conventional therapeutics protocols, more with those from poor socio-economic background. ${ }^{20}$ Along with cancer-associated stress, mental agony and physiological distress considerably multiplied even for cancer patients who are/were COVID negative, more for elderly, women and juveniles. ${ }^{21-26}$ The stressors were infection fears, long term quarantine duration, boredom, inadequate knowledge and information, financial loss; insufficient food supplies and stigma. ${ }^{27,28}$ This was alarming and demanded appropriate intervention by mental health professionals to deal with. ${ }^{29}$
Thus, this imposed lock-down seemed to have profound adverse influences, with increased suicidal cases, changed behavior patterns and life styles of the peoples. ${ }^{20,24,30,31}$

Assam although with six cancer treatment hospitals, six radiotherapy facilities, eight palliative care centres, nine cancer patient welfare schemes and providing better "set of multidisciplinary services" had patients who sought treatments outside home state. ${ }^{14} \mathrm{~A}$ mental health assessment conducted during this lockdown period to understand COVID-19 pandemic lockdown impact in Assam's population concluded that the level of DASS-21* was quite high in the population of Assam in comparison to the findings obtained during the National Mental Health Survey of India, $2016 .{ }^{29}$ This lockdown thus seemed to impose tension on the cancer patients who were in Assam and required to avail treatments either inside or outside the state. In such a case, decisions regarding regular cancer therapy initiations became increasingly complex and the oncology community faced unprecedented challenges because of the sudden lockdown. Many non-COVID hospitals were reluctant to take any new cases of new patients ranging from heart disease, lung kidney problems, diabetes, COPD and even minor ailments in the fear of acquiring COVID-19 infection. ${ }^{32}$ Under these conditions, the medical ethics team and the Oncologist relied on three vital points for management of cancer as well as patients with co morbidities- (i) Non-abandonment of a patients who were dependent on investigational treatment (ii) Make an effort on flattening the COVID-19 curve by minimizing unnecessary exposure to suspicious environment (iii) To emphasize on psychosocial support to the patient amidst this outbreak. ${ }^{33}$ A significant concern and a mammoth challenge were thus managing patients during this crisis. With OPD (outpatient departments) closed and transportation under seize, it was a staggering task to carter the medical, psychological and financial need of these patients. The effects were even more in NE India, which otherwise has one of the country least per capita income and febrile job opportunities. Most, patients hailed from families who were engaged in unorganized sector, and sudden lockdown affected them economically. Not only that nationwide lockdown resulted in economics downfall, leading to job loss and massive unemployment, a mass exodus of migrant labor workforce

\begin{tabular}{|c|c|c|c|c|c|c|c|c|}
\hline & \multicolumn{4}{|c|}{ NCRP (2006-2008) } & \multicolumn{4}{|c|}{ NCRP -2020 (2012-2016 } \\
\hline & Male & Female & Total & $\begin{array}{c}\% \text { of Cancer patients } \\
\text { in NE States }\end{array}$ & Male & Female & Total & $\begin{array}{c}\% \text { of Cancer patients } \\
\text { in NE States }\end{array}$ \\
\hline Rest of India & 72,830 & 70,097 & 142,927 & $13 \%$ & 171,594 & 176,289 & 347,883 & $20 \%$ \\
\hline NE States & 9,399 & 8,704 & 18,103 & & 36,818 & 32,450 & 69,268 & \\
\hline
\end{tabular}


was also witnessed as a fall out of this effect .It also resulted in colossal effect on healthcare facilities as well. To address these problems during this long term quarantine as well as lock down period the specific points were important to be implemented as described by Brooks et al in details ${ }^{20}$ :-

- Information is the key; quarantined people need to understand the importance of the situation, and their family members/neighbors need to be educated about the taboos related to the fear of infection through scientific outlook.

- Communication is essential which requires to be rapid and effective

- Both general and medical supplies need to be provided

- Most of the adverse effects come from the imposition of a restriction of liberty; voluntary quarantine is associated with less distress

- Public health officials should emphasize the altruistic choice of self-isolating.

Faced with these uncertainties, many Non Government Organizations (NGOs) came forward to join hands with the government to fight through these situations. Even, a pilot project entitled, "COVID-19-Sentinel" was initiated. The collaboration was in between an NGO service called Pratishruti Cancer and Palliative Trust and Assam Police to monitor the quarantined population including Cancer patients in Assam's Dhemaji District. A team of 140 volunteers from qualified backgrounds were associated with this initiative. Proper training was provided to the volunteers regarding both crisis intervention skills and COVID-19 pandemic related knowledge. This service was initiated based mainly on online and hotline service protocols for both psychological and health related medical services to be provided public emergency interventions. ${ }^{27,34}$ Even, helpline numbers were launched and published by MyGovAssam (government of Assam) in its social media handles (https://twitter.com/mygovassam/ status/1245544420130189313) for wider dissemination to the public where the dos and don'ts for cancer patients at home and people in general amid the current lockdown due to COVID-19 pandemic were spelled out. ${ }^{32}$

The main agenda and doctrines of the groups were:

- Public participations to address large affected populations;

- Public awareness about the expected psychological impact and reactions to trauma

- Digital Platforms and web based approach to address mental health and psychiatric support (Pratishruti Telemedicine Group and Kavi-Krishna Translational Medicine Centers - for both Quarantine as well as Cancer Patients). The mode of communication for telemedicine was conducted by various apps, video on chat platform skype/face time etc.), audio (phone,
VOIP, apps etc.) and text based (WhatsApp, Google hangouts, Facebook Messenger, Asynchronous like email/fax etc.). Red Cross Society, Helping Hands foundation for food and medicinal supply.

- About 1500 quarantined peoples along with their family members were monitored during this lockdown with significant and positive outcome and active participation from local health care providers and ASHA* workers who actively participated in this crisis. Seeing this success, a similar protocol is now implemented in other $\mathrm{NE}$ Indian districts engaging the local populations to support the cancer patients in palliative and psychological care along with other ailments.

* ASHA:- (accredited social health activist) are community health care workers instituted by the government of India's Ministry of Health and Family Welfare (MoHFW) as a part of the National Rural Health Mission (NRHM) serving as volunteers

- Patients were supervised through Telemedicine by Pratishruti and Kavi Krishna Telemedicine center. Special care was taken for Cancer patients, for their proper regular treatment protocols.

- The protocol followed for the COVID-19 monitoring was:

(a) Migrants travelling from different states by different travel modes were taken to COVID Isolation centers followed by proper verification.

(b) Preliminary examination was followed by nasopharangyl swab collection following which subjects were sent either to Institutional Quarantine centers or given home quarantine depending on circumstances of travel..

(c) A list of the subjects whose samples had been collected was sent to the IT sector of Police Head Quarter under the surveillance unit of COVID-19 Monitoring Panel.

(d) IT sector distributed the list to Human Resource (HR) section after proper verification. The lists were further subdivided amongst ten volunteers under each HR. Each Quarantine person was given a specific code to maintain their privacy.

(e) Prior to list distribution online meetings were being held by the HR team to synchronize the list distribution and choose their team of volunteers. Each HR was provided with two team leaders and 10 volunteers in each.

(f) The Quarantined Persons were kept under continuous surveillance by the volunteers through video or telephonic conversations. Proper advice about Quarantine, Sanitization and Health were given to them. For stress and anxiety relief they were advised to perform some indoor co-curricular 
activities if possible. In case of any stress or anxiety they were expected to call the volunteers.

(g) Within 48 hours the reports were sent to the respective Quarantined people through Smart Messaging Service (SMS). COVID positive subjects were sent to Hospital Quarantine. Amongst those with potential and life threatening co-morbidities like COPD, uncontrolled diabetes, asthma, chronic kidney disease and cancer shifted were shifted to tertiary health care facility*.

* tertiary heath care facility:- highly specialized medical care usually over an extended period of time that involves advanced and complex procedures and treatments performed by medical specialists in state-of-the-art facilities usually for inpatients and on referral from a primary or secondary health professional.

(h) After completion of Quarantine the home quarantined people were asked to make a call to their nearby police station or their respective volunteers as a record.

(i) Emergency Helpline numbers were issued which could be contacted at any time round the clock..

(j) The Psychological and Psychiatric Specialist team readily reached out to any persons in times of need on an emergency basis.

The protocol has been described in Figure 1.

Protocol for management of stress related issues in quarantined people

(a) Quarantine being the restriction and inhibition of movement of people who have been exposed to contagious infection to ascertain their health wellness and compliance with health policy rules, further reducing the risk of infecting others as well. ${ }^{35}$ Studies showed that longer durations of quarantine were associated with post-traumatic stress symptoms, poor mental health, avoidance behaviors and anger related issues as well. ${ }^{36,37}$ Those quarantined were found to 'be experiencing physical symptoms related to this pandemic that initiated several psychological setbacks. The confinement during quarantine led to loss of usual daily routine with reduced physical and social contact with others had impacts like frustration, a sense of isolation from the rest of the world that increased distress among the quarantined people. Further, there was an exacerbation among this group because of not being able to take part in day-today services for basic necessities. ${ }^{37}$ To help these quarantined peoples, the following initiatives were taken:-Quarantined People with stress associated issues were addressed directly by the Team Leaders who were from Health Professional backgrounds (psychiatrist,
Oncologists, Radiotherapists, Medicine-specialists, Endocrinologists, Psychologist, and Surgeons etc).

(b) Special care was taken for Cancer patients with other ailments. In case of Cancer patients, responsibility was given to Pratishruti Cancer and Palliative Trust. With their well equipped medical and para-medical team they provided ready to move in service. This group made video calls to respective specialist who went through the case thereby providing consultation of medication as well as preliminary help provided under proper sanitization protocols. It has been described in details in Figure 2.

(c) The home Quarantined people in distant rural areas were provided with food and medicines in case of scarcity by NGOs like Helping Hands Volunteers as well as North East Police. Govt. Of Assam provided Helpline numbers for providing of specific medicines for Quarantined people. In case of unavailability the respective Volunteers took in charge and managed the gap by providing the necessary amendments to the people in need.

(d) Efforts were made that the Government aided schemesAtal Amrit Abhiyan and Ayushman Bharat could be continued without any hindrance, as this would greatly offer financial assurance to cancer patients during the time of hardship due to the sudden lockdown.

This lockdown and pandemic brought to surface the hidden mental health fears and issues which were left untreated. But, proper counseling and various innovative approaches were introduced to help people cope up with their depressive states through electronic media and under proper social distancing. Volunteers helped the quarantined people by introducing them to various indoor interactive activities like, proper day to day routine followed by yoga and meditation. Extracurricular activities like painting, reading were introduced and if required proper counseling by trained professionals were also provided to them. Thus, the psychological impact of Quarantine has been found to be wide-ranging, with long term adverse mental effects. Necessary measures were and in the near future are important to be taken so that these mental feeds could be handled profoundly. Further, the sudden lock down brought the crisis of food and medicine for normal people as well as for patients with various ailments. This challenge was overcome by dedicated volunteers from different NGOS who risked their lives to help. The major concern and one of the reasons of psychological stress during this lockdown was inadequate basic supplies (e.g.:- food, water, clothes or accommodations), since maximum people who were below the poverty range are daily wagers. ${ }^{38}$ This source of frustration associated with anxiety and anger is still being continued in some cases related to this issue. Moreover, the inadequacy of regular medical care and prescriptions also increased problem in some Quarantined people. ${ }^{20}$ To help these people distribution of food provided by the Government in collaboration with medical health practitioners and various local NGO's was 


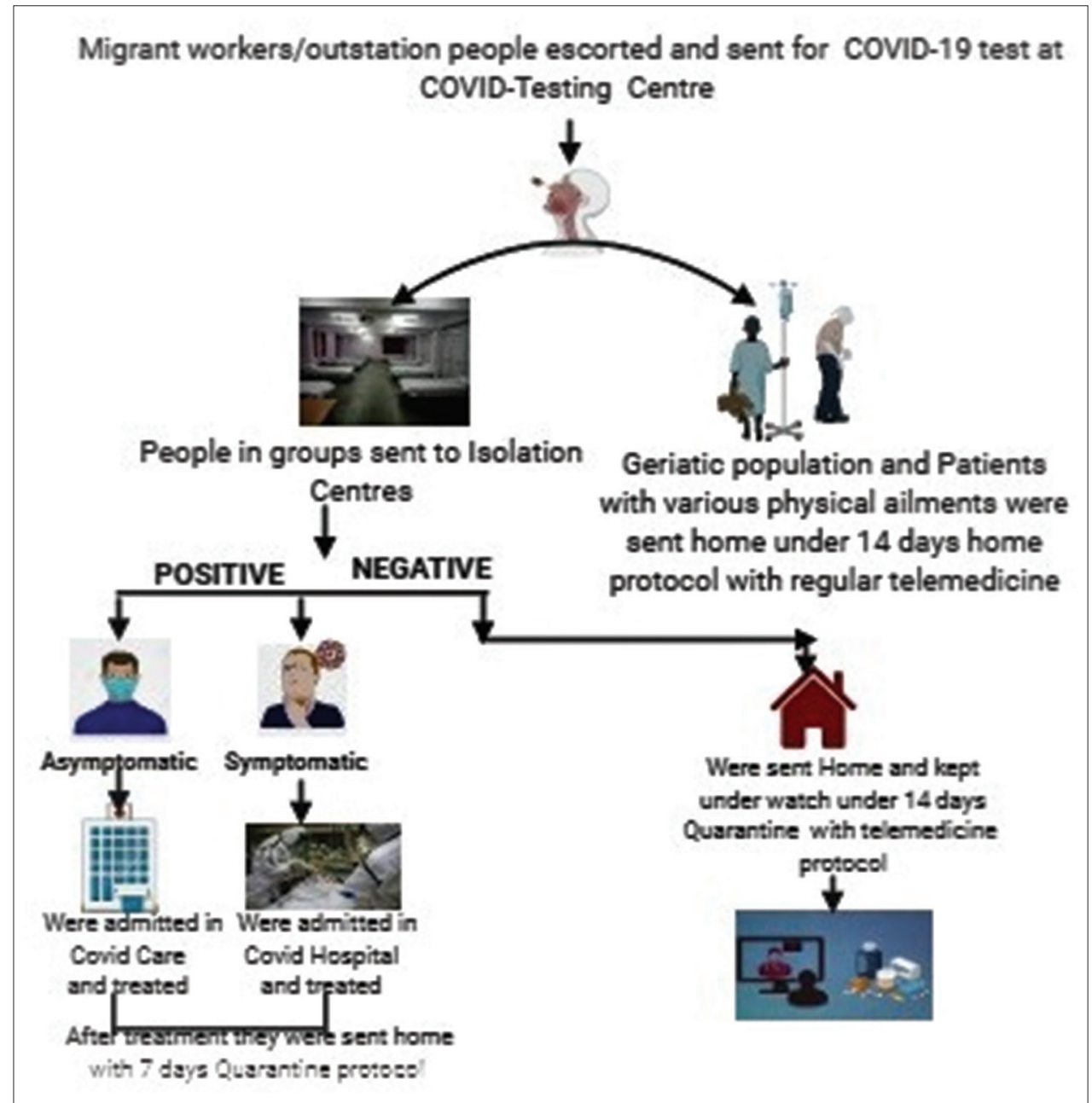

Figure 1: Protocol followed in COVID-19 Sentinel by the NGO's through Telemedine protocol to reach out to the people in Quarantine Geriatic:- Doctor who diagnosis and treat age related health condition

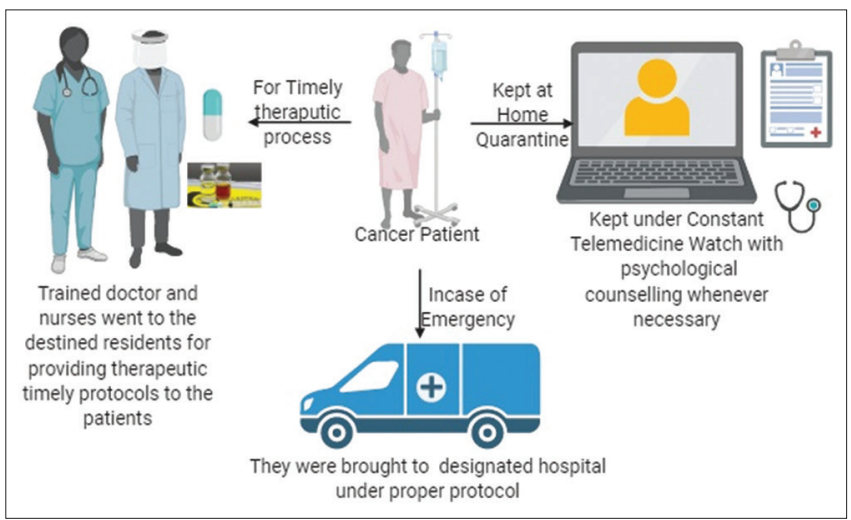

Figure 2: Protocol followed through Telemedine to help the Cancer Patients in Rural Sections during the period of lockdown to continue with their regular treatments

done almost after every 15 days following proper sanitization and social distancing protocols. Telemedicine that literally means "healing at a distance" was being used as one of the very useful tools of communication between the doctor and the patient. ${ }^{39}$ Das et al., from Dr B. Borooah Cancer
Institute, Guwahati, Assam, India aimed to conduct a study in the utility of telemedicine during the lockdown period of COVID-19 pandemic in North East India. The team made a cross-sectional study among cancer patients present at their center on follow up or ongoing treatment. Analysis of all the data acquired from telephonic conversation with their patients from $30^{\text {th }}$ March, 2020 to $3^{\text {rd }}$ May, 2020. The study concluded that telemedicine although can't replace conventional method of in treatment, but it proved to be a useful tool during the COVID-19 pandemic for patient follow up and treatment of cancer patients. ${ }^{40}$ In a country like India, with more than 1.36 billion population where the doctor: patient ratio is 1:1456 the providing of health care has been a challenge and that to in North east India where communication is added to the above challenges. ${ }^{40}$ One of the big advantages of the telemedicine protocol followed was for the people in rural India, which reduced the cost and logistic issues to reach out specialist doctor. Telemedicine was able to cut down the financial cost of consultation as well as inconvenience of troublesome travel for obtaining referral services. ${ }^{41-43}$ Further, this assurance of basic supplies (such as food, water, 
and medical supplies) when made available that reinforced the sense of altruism amongst those terrified people. ${ }^{20}$ As such, during this Covid-19 Pandemic the dedicated NGO's worked restlessly and served as a backbone of the entire health professional team and Government. Thus, these NGOs and their volunteers were able to build bridge and fill the gap by providing necessary aids to Quarantined as well as normal people whenever necessary. Its goes without saying the participations of NGOs to fill the gaps makes all the differences between life and death in these periods of crisis when the healthcare infrastructure and logistics are at stake. NGO with their relentless selfless efforts to support the patients as well as people during COVID times is remarkable and this effort can surely be credited for low mortality rates in Assam even under such an adverse crisis.

\section{Abbreviations}

* ARDS:- Acute Respiratory Distress Syndrome

* PBCR:- Population Based Cancer Registry

* DASS-21:- Depression, Anxiety and Stress Scale (DASS-21)

* ASHA:- Accredited social health activist.

* COPD:- Chronic Obstructive Pulmonary Disease

\section{REFERENCES}

1. Sharma JD, Kalit M, Nirmolia T, Saikia SP, Sharma A and Barman D. Cancer: scenario and relationship of different geographical areas of the globe with special reference to North East-India. Asian Pac J Cancer Prev. 2014;15(8):3721-3729.

https://doi.org/10.7314/APJCP.2014.15.8.3721

2. India State-Level Disease Burden Initiative Cancer C. The burden of cancers and their variations across the states of India: the Global Burden of Disease Study 1990-2016. Lancet Oncol. 2018;19(10):1289-1306.

https://doi.org/10.1016/S1470-2045(18)30447-9

3. Kumar R, Rai AK, Das D, Das R, Kumar RS, Sarma A, et al. Alcohol and Tobacco Increases Risk of High Risk HPV Infection in Head and Neck Cancer Patients: Study from North-East Region of India. PLoS One. 2015;10(10):e0140700 https://doi.org/10.1371/journal.pone.0140700

4. Liang W, Guan W, Chen R, Wang W, Li J, Xu K, et al. Cancer patients in SARS-CoV-2 infection: a nationwide analysis in China. Lancet Oncol. 2020;21(3):335-337.

https://doi.org/10.1016/S1470-2045(20)30096-6

5. Kuderer NM, Choueiri TK, Shah DP, Shyr Y, Rubinstein SM, Rivera DR, et al. Clinical impact of COVID-19 on patients with cancer (CCC19): a cohort study. Lancet. 2020;395(10241):1907-1918. https://doi.org/10.1016/S0140-6736(20)31187-9

6. Song K, Gong H, Xu B, Dong X, Li L, Hu W, et al. Association between recent oncologic treatment and mortality among patients with carcinoma who are hospitalized with COVID-19: A multicenter study. Cancer. 2020.

https://doi.org/10.1002/cncr.33240

7. Vellas C, Delobel P, de Souto Barreto P and Izopet J. COVID-19, Virology and Geroscience: A Perspective. J Nutr Health Aging. 2020;24(7):685-691.

https://doi.org/10.1007/s12603-020-1416-2
8. Pal R and Yadav U. COVID-19 Pandemic in India: Present Scenario and a Steep Climb Ahead. J Prim Care Community Health. 2020;11:2150132720939402. https://doi.org/10.1177/2150132720939402

9. Miyashita H, Mikami T, Chopra N, Yamada T, Chernyavsky S, Rizk D, et al. Do patients with cancer have a poorer prognosis of COVID-19? An experience in New York City. Ann Oncol. 2020;31(8):1088-1089.

https://doi.org/10.1016/j.annonc.2020.04.006

10. WHO. World Health Statistics 2019: Monitoring Health for the SDGs,sustainable development goals.. Organization WH, editor. Geneva, Switzerland,2019. https://www.who.int/publications/i/ item/world-health-statistics-2019-monitoring-health-for-thesdgs-sustainable-development-goals

11. Mathur P, Sathishkumar K, Chaturvedi M, Das $P$, Sudarshan $K L$, Santhappan S, et al. Cancer Statistics, 2020: Report From National Cancer Registry Programme, India. JCO Glob Oncol. 2020;6:1063-1075. https://doi.org/10.1200/GO.20.00122

12. Programme NCR. Three year report of population based cancer registries 2006-08, national cancer registry programme. In: Indian council of medical research I, editor.: Indian council of medical research, India; 2010. https://ncdirindia.org/ncrp/ Annual_Reports.aspx

13. NCDIR, Indian Council of Medical Research. Annual report (2016-17) WWW page. http://www.ncdirindia.org

14. Ngaihte P, Zomawia E and Kaushik I. Cancer in the NorthEast India: Where we are and what needs to be done? Indian J Public Health. 2019;63(3):251-253. https://doi.org/10.4103/ijph.IJPH_323_18

15. National cancer registry programme. Three-year report of population based cancer registries 2012-2014 WWW page. 2016. http://ncdirindia.org

16. Bootsma MC and Ferguson NM. The effect of public health measures on the 1918 influenza pandemic in U.S. cities. Proc Natl Acad Sci U S A. 2007;104(18):7588-7593.

https://doi.org/10.1073/pnas.0611071104

17. lezadi S, Azami-Aghdash S, Ghiasi A, Rezapour A, Pourasghari $\mathrm{H}$, Pashazadeh $\mathrm{F}$, et al. Effectiveness of the nonpharmaceutical public health interventions against COVID-19; a protocol of a systematic review and realist review. PLoS One. 2020;15(9):e0239554.

https://doi.org/10.1371/journal.pone.0239554

18. Neil M, Ferguson DL, Gemma Nedjati-Gilani, Imai N, Ainslie K, Baguelin $\mathrm{M}$, et al. Report 9: Impact of non-pharmaceutical interventions (NPIs) to reduce COVID-19 mortality and healthcare demand. Imperial College,London: Imperial College,London; 202016 March,2020. Report No: 9.https://www.imperial.ac.uk

19. Kutikov A, Weinberg DS, Edelman MJ, Horwitz EM, Uzzo RG and Fisher RI. A War on Two Fronts: Cancer Care in the Time of COVID-19. Ann Intern Med. 2020;172(11):756-8. https://doi.org/10.7326/M20-1133

20. Brooks SK, Webster RK, Smith LE, Woodland L, Wessely S, Greenberg N, et al. The psychological impact of quarantine and how to reduce it: rapid review of the evidence. Lancet. 2020;395(10227):912-920. https://doi.org/10.1016/S0140-6736(20)30460-8

21. Castelnuovo G, De Giorgio A, Manzoni GM, Treadway DC and Mohiyeddini C. Psychological, Behavioral, and Interpersonal Effects and Clinical Implications for Health Systems of the Coronavirus (COVID-19) Pandemic: A Call for Research. Front Psychol. 2020;11:2146.

https://doi.org/10.3389/fpsyg.2020.02146 
22. Rodriguez-Rey R, Garrido-Hernansaiz H and Collado S. Psychological impact of COVID-19 in Spain: Early data report. Psychol Trauma. 2020;12(5):550-552.

https://doi.org/10.1037/tra0000943

23. Guessoum SB, Lachal J, Radjack R, Carretier E, Minassian S, Benoit L, et al. Adolescent psychiatric disorders during the COVID-19 pandemic and lockdown. Psychiatry Res. 2020;291:113264

https://doi.org/10.1016/j.psychres.2020.113264

24. Gunnell D, Appleby L, Arensman E, Hawton K, John A, Kapur N et al. Suicide risk and prevention during the COVID-19 pandemic. Lancet Psychiatry. 2020;7(6):468-471.

https://doi.org/10.1016/S2215-0366(20)30171-1

25. Commodari E and La Rosa VL. Adolescents in Quarantine During COVID-19 Pandemic in Italy: Perceived Health Risk, Beliefs, Psychological Experiences and Expectations for the Future. Front Psychol. 2020;11:559951.

https://doi.org/10.3389/fpsyg.2020.559951

26. Castelli L, Castelnuovo G and Torta R. Editorial: PsychOncology: clinical psychology for cancer patients-Cancer: the key role of clinical psychology. Front Psychol. 2015;6:947.

https://doi.org/10.3389/fpsyg.2015.00947

27. Wang $\mathrm{J}$, Wei $\mathrm{H}$ and Zhou L. Hotline services in China during COVID-19 pandemic. J Affect Disord. 2020;275:125-126.

https://doi.org/10.1016/j.jad.2020.06.030

28. Kochhar AS, Bhasin R, Kochhar GK, Dadlani H, Mehta VV Kaur $\mathrm{R}$, et al. Lockdown of 1.3 billion people in India during Covid-19 pandemic: A survey of its impact on mental health. Asian J Psychiatr. 2020;54:102213.

https://doi.org/10.1016/j.ajp.2020.102213

29. Gogoi AJ and Sarmah C. COVID-19 and Lockdown: Impact on Mental Health Among the Residents of Assam, India. Asia Pac J Public Health. 2020:1010539520962952.

https://doi.org/10.1177/1010539520962952

30. Goyal K, Chauhan P, Chhikara K, Gupta P and Singh MP. Fear of COVID 2019: First suicidal case in India ! Asian J Psychiatr. 2020;49:101989.

https://doi.org/10.1016/j.ajp.2020.101989

31. Mamun MA and Griffiths MD. First COVID-19 suicide case in Bangladesh due to fear of COVID-19 and xenophobia: Possible suicide prevention strategies. Asian J Psychiatr. 2020;51:102073. https://doi.org/10.1016/j.ajp.2020.102073

32. Kataki AC and Krishnatreya M. Cancer care and COVID-19 pandemic: An experience from cancer center in North-East India.. Curr Med Issues. 2020 18(3):4.

https://doi.org/10.4103/cmi.cmi_100_20
33. Shuman AG and Pentz RD. Cancer Research Ethics and COVID-19. Oncologist. 2020.

https://doi.org/10.1634/theoncologist.2020-0221

34. Liu S, Yang L, Zhang C, Xiang YT, Liu Z, Hu S, et al. Online mental health services in China during the COVID-19 outbreak. Lancet Psychiatry. 2020;7(4):e17-e18. https://doi.org/10.1016/S2215-0366(20)30077-8

35. Quarantine and Isolation. Centre for Disease Control and Prevention. 2017 https://www.cdc.gov/quarantine/index.html

36. Hawryluck L, Gold WL, Robinson S, Pogorski S, Galea S and Styra R. SARS control and psychological effects of quarantine, Toronto, Canada. Emerg Infect Dis. 2004;10(7):1206-1212. https://doi.org/10.3201/eid1007.030703

37. Reynolds DL, Garay JR, Deamond SL, Moran MK, Gold W and Styra R. Understanding, compliance and psychological impact of the SARS quarantine experience. Epidemiol Infect. 2008;136(7):997-1007. https://doi.org/10.1017/S0950268807009156

38. Wilken JA, Pordell P, Goode B, Jarteh R, Miller Z, Saygar BG, et al. Knowledge, Attitudes, and Practices among Members of Households Actively Monitored or Quarantined to Prevent Transmission of Ebola Virus Disease - Margibi County, Liberia: February-March 2015. Prehosp Disaster Med. 2017;32(6):673-678. https://doi.org/10.1017/S1049023X17006720

39. Strehle EM and Shabde N. One hundred years of telemedicine: does this new technology have a place in paediatrics? Arch Dis Child. 2006;91(12):956-959.

https://doi.org/10.1136/adc.2006.099622

40. Associated Press. Doctor-patient ratio in India less than WHOprescribed norm of 1:1000: Govt. The Economic Times. 2019 Nov 20. https://health.economictimes.indiatimes.com/news/ industry/doctor-patient-ratio-in-india-less-than-who-prescribednorm-of-11000-govt/72135237

41. Das AK, Boro SS, Das A, Medhi P, Kakati K, Baishya N, et al. Utility of telemedicine in COVID-19 pandemic: our experience at a tertiary cancer center in North East India. International Journal of Research in Medical Sciences. 2020;8(11):4027-4030. https://doi.org/10.18203/2320-6012.ijrms20204898

42. Monaghesh $E$ and Hajizadeh $A$. The role of telehealth during COVID-19 outbreak: a systematic review based on current evidence. BMC Public Health. 2020;20(1):1193. https://doi.org/10.1186/s12889-020-09301-4

43. Das AK, Boro SS, Das A, Medhi PP, Kakati K, Baishya N, et al. Utility of telemedicine in COVID-19 pandemic: our experience at a tertiary cancer center in North East India. Int J Res Med Sci. 2020 ;8(11):4027-4030.

https://doi.org/10.18203/2320-6012.ijrms20204898

\footnotetext{
Author's Contributions:

JT \& SK-Conceptualized and designed the study, literature search, prepared first draft of the manuscript, critical revision of the manuscript;

AK \& Arun K-Critical revision of the manuscript, diagrammatic conceptualization of the review; RD-Concept of the study, literature search, review of the study; SK-Overseen the entire work.

Work Attributed to:

Department of Biochemistry, AlIMS, New Delhi.

Orcid ID:

Dr. Joyeeta Talukdar- (1) https://orcid.org/0000-0002-4922-2283

Abhibroto Karmakar- (1) https://orcid.org/0000-0002-9697-9107

Dr. Ruby Dhar- (D) https://orcid.org/0000-0003-3600-6554

Dr. Arun Kumar- (i) http://orcid.org/0000-0002-8800-0296

Dr. Subhradip Karmakar- io https://orcid.org/0000-0002-4757-8729

Source of funding: None, Conflicts of Interest: The authors declare that the study was conducted in the absence of any commercial or financial relationships that could pose as a potential conflict of interest.
} 